\title{
Heinz Bude
}

\section{Die 50er Jahre im Spiegel der Flakhelfer- und der 68er-Generation}

Man kann Deutschland als das Land der Generationen bezeichnen - jedenfalls im Unterschied zu Großbritannien, das bis heute ein Land der Klassen ist, oder zu Frankreich, dessen Selbstverständnis auf der Idee der Republik gründet. Dabei geht es natürlich nicht um die Tatsachenfrage, ob es nicht auch in Frankreich Klassen und in Großbritannien Generationen gibt oder ob Deutschland nicht auch eine Republik darstellt, sondern um den herrschenden Orientierungsbegriff für die politische Kultur eines Landes, auf den sich die kontroversen Vorstellungen der gesellschaftlichen Selbstthematisierung beziehen. Darin liegt der Unterschied. So werden bei uns gesamtgesellschaftliche Zäsuren ganz selbstverständlich mit Generationswechseln in Verbindung gebracht $\left({ } 1914^{\star}\right.$ oder ${ }_{n} 1968^{\alpha}$ zum Beispiel) ${ }^{1}-$ oder es werden soziokulturelle Staus mit der überlangen Dominanz einer nicht abtreten wollenden Generation erklärt (die "Wilhelminer des Kaiserreichs" am Anfang $^{2}$ und die "Skeptiker der Bundesrepublik" am Ende des 20. Jahrhunderts ${ }^{3}$ ).

Das Phänomen der Generationen allerdings ist international und modern. Die am Beginn des 20. Jahrhunderts stehende "Generation von $1914^{44}$ gab es in Großbritannien und in Frankreich genauso wie in Spanien, Italien und Deutschland. Sicher mit anderen Ausdrucksformen - die Briten schrieben Gedichte, Briefe und Novellen, die Italiener politische Essays, die Spanier hörten auf die Vorlesungen eines Ortega, die Franzosen liebten das kollektive Portrait einer jungen intellektuellen Pariser Elite, und die Deutschen schrieben groß angelegte soziale Theorien -, aber das Thema war immer dasselbe: Abschied von einem langweiligen, friedlichen, selbstzweifelnden und glaubenslosen 19. Jahrhundert und die Hinwendung zu einer nationalistischen, futuristischen und militanten Moderne. Der Krieg war für die "Generation von 1914" zu einer Frage des Lebens geworden.

In diesem säkularen Bruch setzte Deutschland allerdings einen spezifischen Akzent. Um die Wende vom 19. aufs 20. Jahrhundert taucht hier der Begriff der

1 Martin Broszat (Hrsg.), Zäsuren nach 1945. Essays zur Periodisierung der deutschen Nachkriegsgeschichte (München 1990).

2 Martin Doerry, Übergangsmenschen (Weinheim 1989).

${ }^{3}$ Heinz Bude, Die Politik der Generationen (Gewerkschaftliche Monatshefte 49, Opladen 1998) 689-694.

4 Robert Wobl, The Generation of 1914 (Cambridge 1979). 
"Jugendgeneration" auf, womit zum Ausdruck gebracht wird, wie Jugend und Generation zu austauschbaren Begriffen werden. ${ }_{n}$ Jugend " bezeichnet das Recht zum absoluten Neuanfang und "Generation" die Geworfenheit in eine ganz und gar einmalige geschichtliche Situation. Weil niemand sonst die Einwirkungen und Forderungen der Jetztzeit in dieser Frische und Entschiedenheit erleben kann, kann sich die „junge Generation“ als Vorhut einer neuen Zeit fühlen ${ }^{5}$.

Diese aus Deutschland kommende semantische Innovation veranschaulicht die drei Gründe für die Modernität des Phänomens der Generation:

Da ist zuerst ein ideeller Komplex, der die Botschaft der Französischen Revolution mit dem Programm der deutschen Romantik verbindet ${ }^{6}$. Wo das revolutionäre Prinzip besagt, daß Vergangenheit zugunsten von Zukunft negierbar ist, behauptet die aus dem Zusammenbruch der Systemphilosophien stammende Denkbewegung den Primat des Lebens und der Existenz gegenüber dem Geist und der Geschichte. Im Generationenbegriff läßt sich die äußerste Mobilisierung von Zukunft mit dem "unendlichen Mangel an Sein" (Schelling) zusammendenken. Denn die Kraft zur Unterbrechung der Geschichte kommt aus der Rückkehr zum Wirklichen und Konkreten?.

Diese Ideen bedürfen allerdings bestimmter Institutionen für ihre Formierung und Verbreitung. So beruht der Auftritt von Generationen als eigenständigen Akteursfiktionen auf der institutionellen Durchsetzung von Schule und Wehrpflicht als Agenturen für die Herstellung altershomogener Gruppen mit einem eigenen Selbstverständnis ${ }^{8}$. Durch schulische Unterweisung und militärische Disziplin entsteht die männliche Gruppe der Gleichaltrigen, die als Jugend ihr eigenes Recht fordert. Deshalb ist für die klassische Generationentheorie diese eigene fürs Experimentieren freigegebene Phase im Lebenslauf so wichtig für die Bildung eines generationellen Selbstverständnisses.

Neben diesen Ideen und Institutionen waren bestimmte Interessen für die Durchsetzung des Generationenbegriffs verantwortlich. Dahinter stand die besonders in Deutschland um die Jahrhundertwende auftretende demographische Revolution, die den Jungen das Gefühl robuster Anspruchsberechtigung allein schon aufgrund zahlenmäßiger Stärke vermittelte?. Weil sich die Idee des Neuen nicht nur mit der Erfahrung gleichartiger Einwirkungen, sondern auch noch mit dem Gefühl gemeinsamer Interessen verband, konnte Generation im 20. Jahrhundert ein ebenso starker Mobilisierungsbegriff werden wie Klasse und Nation im 19. Jahrhundert.

5 Thomas Koebner, Rolf-Peter Janz, Frank Trommler (Hrsg.), „Mit uns zieht die neue Zeit“. Der Mythos Jugend (Frankfurt a.M. 1985).

6 Pierre Nora, Generation, in: ders. (ed.), Realms of Memory, Vol.1: Conflicts and Divisions (New York 1996) 499-531.

7 Marvin Rintala, The Constitution of Silence. Essays on Generational Themes (Westport, London 1979).

${ }^{8}$ Friedrich H. Tenbruck, Jugend und Gesellschaft (Freiburg $\left.{ }^{2} 1965\right)$.

${ }^{9}$ Detlev Peukert, Die Weimarer Republik (Frankfurt a. M. ${ }^{51996) .}$ 
Besondere Entstehungskonstellationen ändern allerdings nichts an der Globalität des Phänomens. Sobald eine Generation eine identifizierbare Gestalt annimmt, findet sie sich immer in mehreren Ländern zugleich. Das ist nicht erst seit der weltweiten Bewegung von 1968 der Fall. Schon die erste nachrevolutionäre Generation der Romantik war zumindest ein europäisches Phänomen ${ }^{10}$. Das läßt sich genauso wie für die „Generation von 1914“, für die Generation der politischen Jugend aus der Zwischenkriegszeit, für die skeptische Generation der Nachkriegszeit, selbst für die "Halbstarken“ zeigen, die zwischen 1956 und 1958 mit Bill Hailey und James Dean außer Rand und Band gerieten. Die hießen in Großbritannien "Teddy-Boys“, in Dänemark „Laeder-Jakken“, in Spanien „Gaberros“, in Österreich "Plattenbrüder", in Frankreich "Blouson Noir“ und in der Sowjetunion ${ }_{n}$ Hooligans ${ }^{\star 11}$. Es geht jedesmal um die Erfahrung überraschender Übereinstimmungen $z$ wischen Ausdrucksformen und Problemwahrnehmungen, die sich nicht auf gegenseitige Abhängigkeiten zurückführen lassen und die trotzdem die Grenzen der einzelnen Gesellschaften überschreiten. Es ist die Bezugnahme auf ein sozialisierendes Eindrucks- und Wirkungserlebnis, aus dem sich die Evidenz einer Gemeinsamkeit der geschichtlichen Lage trotz erkennbarer Unterschiede in sozialer Herkunft und nationalem Ursprung ergibt. Unbeschadet der Tatsache, daß die Einarbeitung in die jeweilige Gesellschaftsgeschichte in ganz unterschiedlicher Weise verläuft, stellen Generationen die ersten Globalisierungsvorgänge dar, die quer zu mehreren Gesellschaften und über sie hinweg verlaufen.

Es hängt sicher mit den bekannten „Verspätungen“ und „Sonderwegen “ unserer Gesellschaftsgeschichte zusammen, daß in Deutschland Fragen des kollektiven Selbstverständnisses mit Vorliebe an Generationskonflikten festgemacht werden. Der „verspäteten Nation“ fehlt ein "goldenes Zeitalter“, das einen mythischen Grund und einen traditionsstiftenden Bezugspunkt ihres Selbstverständnisses abgeben könnte ${ }^{12}$. Wir hatten keine große Revolution und kennen keine konsolidierte Periode in unserer Geschichte. Wo keine Tradition lebendig ist, muß man sich auf die vergehende Zeit selbst beziehen. Deshalb sind die Deutschen zum Volk der Geschichte geworden, das das „historische Bewußtsein " erfunden hat und deshalb seine Geschichte weniger nach dem Stand der Klassenkämpfe oder den Formen der Republik, sondern nach Generationen und Generationsverhältnissen periodisiert.

Dabei bilden Generationen ein eigenartig „übertriebenes $W_{i 1}{ }^{* 13}$. Sie gründen zwar in den biographischen Erfahrungen des Ichs, zeichnen aber von seinen varia-

10 Alan B. Spitzer, The French Generation of 1820 (Princeton 1987).

11 Thomas Grotum, Die Halbstarken. Zur Geschichte einer Subkultur in den 50er Jahren (Frankfurr a. M., New York 1994).

12 Darin sind sich zwei prominente Biographen der deutschen Nation einig: nämlich $\mathrm{Hel}$ muth Plessner, Die verspätete Nation (Stuttgart ${ }^{2} 1959$ ) und Norbert Elias, Studien über die Deutschen. Machtkämpfe und Habitusentwicklung im 19. und 20. Jahrhundert (Frankfurt a.M. 1989).

13 Julia Kristeva, Eine Erinnerung (Schreibheft 26, Essen 1985) 134-143. 
blen Verlaufsformen nur ein schematisches Bild. Sie appellieren ans Erlebnis, doch orientieren sich an generellen Vorgaben. Darauf beruht die eigentümliche Orientierungsleistung von Generationen: daß sie die Geschichte unterlaufen und die Biographie steigern.

Das geschieht im Alltag dadurch, daß die Kontingenz der eigenen Biographie in den Vergleichshorizont der Lebensläufe von Gleichaltrigen gestellt wird, woran man sehen kann, was man erreicht hat, woran man gescheitert ist und was bloßes Glück oder Unglück war. Im Vergleichshorizont der Generation findet das Kontingenzerleben der Biographie einen Anker im allgemeinen Geschichtsverlauf. Fragen nach der Generationszugehörigkeit haben mit Gefühlen der Mitgelebtheit, des persönlichen Einsatzes und des gemeinsamen Alterns zu tun. Anders als der Gesellschaftsbegriff, der eine Abstraktion der Perspektive auf eine Sprache von Strukturen, Funktionen und Variationen verlangt, bleibt der Generationsbegriff bei den Wirklichkeiten der menschlichen Erfahrung, die direkter Kommunikation und persönlicher Intuition zugänglich sind. In alltäglichen Unterhaltungen begründen Ergänzungsreaktionen und Themenverschmelzungen plötzlich eine eigentümliche Nähe zwischen ansonsten sich fremd gegenüberstehenden Individuen. Man erkennt sich in einem Gefühl der gleichartigen Betroffenheit durch eine einzigartige gesellschaftliche und geschichtliche Situation.

Dabei macht sich eine bestimmte Pragmatik generationeller Selbstattribuierungen geltend ${ }^{14}$. Da ist einmal der Sachverhalt, daß sich Generationen über Differenzen bestimmen. Man weiß zuerst, zu welcher Generation man nicht gehört. Die Bestimmung der eigenen Generation läuft über Abgrenzungen gegenüber anderen, vorhergehenden oder nachfolgenden Generationen, denen man sich weder stimmungsmäßig, was Vorlieben für Musik, Kleidung oder Gesten betrifft, noch sozialmoralisch, was Ansichten über gesellschaftliche Probleme und kollektive Errungenschaften angeht, zurechnen mag. Bei der Namensnennung für die eigene Generation orientiert man sich an öffentlich gehandelten Vorgaben, die daraufhin geprüft werden, ob sie zur Abgrenzung taugen und das Eigene zu erkennen geben.

Sodann bilden, worauf Karl Mannheim ${ }^{15}$ eindrücklich hingewiesen hat, Generationen polare Einheiten. Es gehört zur Erfahrung von Generationen, daß ungefähr Gleichaltrige zu verschiedenen Folgerungen aus gemeinsamen Erfahrungen und Erinnerungen gelangt sind. In der Regel sind sich die Deutungssender einer Generation nicht einig, welche Schlüsse aus den gemeinsamen Erfahrungen und Erinnerungen zu ziehen sind. Das kann den Charakter eines politischen Streits über konservative und progressive Lesarten des Generationsschicksals haben, aber es kann genauso den Charakter von rein ästhetischen oder völlig stilistischen Kontroversen annehmen. Einig ist man sich freilich im Hinweis auf Momente ${ }_{n}$ er-

14 Heinz Bude, Qualitative Generationsforschung, in: Uwe Flick, Ernst von Kardorff, Ines Steinke (Hrsg.), Qualitative Forschung. Ein Handbuch (Reinbek bei Hamburg 2000) 187-194. 15 Karl Mannbeim, Das Problem der Generationen, in: Karl Mannheim, Wissenssoziologic (Berlin 1964) 509-565. 
habener Rührung“, die einen bestimmten Enthusiasmus des Beginnens oder Auftretens markieren.

Außerdem berührt die generationenmäßige Selbstidentifikation das Verhältnis zwischen avantgardistischen und rezeptiven Gruppen innerhalb eines Generationszusammenhalts. Die Artikulation des Gemeinsamen vollzieht sich über Zeit, das heißt es gibt diejenigen, die die Stichworte liefern und die Embleme verkörpern, und die Mehrheit derjenigen, die sich im Nachhinein darin wiedererkennen und zusammenfühlen. Dies erklärt auch das eigenartige Phänomen der retrospektiven Vermehrung einer Generation. Was am Anfang das Spiel von einigen Wenigen, aber Auffälligen war, bietet im Abstand einen Bezugspunkt für das Selbstverständnis vieler anderer. Man fühlt sich als Angehöriger der Flakhelfer-Generation, obwohl man nie an einem Flak-Geschütz gestanden, oder als Angehöriger der 68er-Generation, obwohl man nie an einer Demonstration teilgenommen hat. Das Zusammengehörigkeitsgefühl bemißt sich nach der Bedeutung, die bestimmte Ereignisse und Situationen haben, und nicht daran, ob man tatsächlich dabei oder wirklich betroffen war ${ }^{16}$.

Schließlich können sogar die typischen Ausdrucksgestalten einer Generation mit der Zeit wechseln. Mannheim hat mit Julius Petersen auf den Wechsel zwischen „führenden“, „umgelenkten “ und „unterdrückten“ Generationstypen bei der Herausarbeitung einer Generationsgestalt hingewiesen. Der Typ der Stunde kann sich schnell als wirre Ausnahmefigur erweisen, der den damals im Hintergrund oder Danebenstehenden nur die Schau gestohlen hat. So durchlaufen Generationen durchaus eine historische Metamorphose, die „umgelenkte Ansichten und "unterdrückte ${ }^{\alpha}$ Strebungen an den Tag bringt. Deshalb muß man sich immer darüber im klaren sein, zu welchem Zeitpunkt man eine Generation charakterisiert, welchen Umfang an Generationsgenossen man zulassen will, wie die Einheit der Gegensätze in einem Generationszusammenhang zu verstehen ist und wie sich die Abgrenzungen zu anderen Generationen mit der Zeit verschieben.

Im Blick auf scine alltägliche Verwendungsweise wird deutlich, wie der Generationsbegriff seine Stellung zwischen dem Geschichts- und dem Gesellschaftsbegriff findet: Gegenüber der „Realabstraktion “ der Gesellschaft behauptet der Generationsbegriff den Erlebnisbezug des Beteiligtseins; und gegenüber der Singularitätsunterstellung des Geschichtsbegriffs besteht der Generationsbegriff auf der „Gleichzeitigkeit des Ungleichzeitigen“ (Wilhelm Pinder). So modalisieren Generationen die Obligation der Gesellschaft und vervielfältigen die Kontinuität der Geschichte. Was wir als Einheit einer gesellschaftsgeschichtlichen Periode ansehen, unterliegt der perspektivischen Variation im Generationenverhältnis. Die geläufige Querschnittsperspektive verdunkelt nur die Widersprüche und Spannungen, die sich in der Längsschnittperspektive ergeben. Was den Geist einer Zeit oder den Begriff einer Epoche ausmacht, ist nur aus dem jeweils geltenden Generationenverhältnis zu klären.

16 Heinz Bude, Die biographische Relevanz der Generation, in: Martin Kobli, Marc Szydlik (Hrsg.), Generationen in Familie und Gesellschaft (Opladen 2000) 19-35. 
Das soll jetzt in bezug auf die 50er Jahre dargelegt werden, die als das Erlebnisjahrzehnt ${ }_{z} \mathrm{z}$ wischen der Abschaffung der Lebensmittelkarten und dem ersten Auftritt der Beatles ${ }^{417}$ bestimmt werden: wie hier Kontinuitäts- und Diskontinuitätsvorstellungen, Traditions- und Modernitätsbegriffe, Restaurations- und Revolutionsaspekte gleichzeitig existieren und sich als $W_{1}$ idersprüche zwischen ungleichzeitigen Generationen reflektieren.

Ich greife dazu auf meine eigenen beiden Untersuchungen über die FlakhelferGeneration und die 68er Generation zurück ${ }^{18}$. Diese beiden Generationen organisieren nämlich in der Retrospektive ihre Lebensgeschichte aus zwei ganz unterschiedlichen Begriffen der 50er Jahre. Was sich für die 68er Kriegskinder als dunkle und enge Zeit von politischer Restauration und persönlichem Erfahrungshunger darstellt, ist für die Flakhelfer, die als „letzte Helden des Führers“ das Ende des Zweiten Weltkriegs erlebt haben, eine helle und offene Zeit der Geschichtsunterbrechung und des Neuanfangs gewesen. In den Interviews knüpfte sich an den Topos der "50er Jahre“ eine nicht-stimulierte Unterschiedsbestimmung, die den Kern des Lebensgefühls der beiden Generationen trifft.

Ich wähle dazu Textausschnitte aus zwei von mir rekonstruierten Fällen: Der 68er Fall ist Carmilla Blisse, die 1940 geboren ist und heute eine der führenden intellektuellen Feministinnen der Bundesrepublik darstellt. Dem Flakhelferfall habe ich den Namen Christoph Westmeyer gegeben, der 1928 geboren ist und zur Zeit der Interviews Professor für Politische Wissenschaften in Aachen war. Beide kann man durchaus als Stichwortgeber ihrer Generation bezeichnen, die schon von ihrem Selbstverständnis her ihrer Biographie einen exemplarischen Anspruch verleihen.

Beginnen wir mit Camilla Blisse.

Können Sie sagen, wann für Sie die SOer Jahre zu Ende waren? Ich meine dieses Gefühl der Enge und der strikten Normalität. Wann das für Sie vorbei war?

Das ist bei mir etwas schwierig zu sagen, weil ich Ende der SOer Jabre angefangen habe zu studieren, 'S 8 habe ich Abitur gemacht und mit dem Studium begonnen, und bin dann weggegangen aus Bethel, aus dieser Anstalt, wo ich zehn Jabre war, und dann fing sowieso ein völlig neues Leben an.

$\mathrm{Mhm}$.

Außerdem, ich war so was von unpolitisch, und wir waren damals alle, die ich kannte, vollkommen unpolitisch.

Ja.

Das ist unglaublich, das kann man sich heute gar nicht mehr vorstellen. Überhaupt keine Ahnung, nicht das geringste Interesse, sich damit $z u$ beschäftigen, was diese Politik eigentlich bedeutet. Ich kann mich nur an die Aufrüstungsdebatte

17 Georg Bollenbeck, Gerhard Kaiser (Hrsg.), Die janusköpfigen 50er Jahre (Wiesbaden 2000) 9.

${ }^{18}$ Heinz Bude, Deutsche Karrieren. Lebenskonstruktionen sozialer Aufsteiger aus der Flakhelfer-Generation (Frankfurt a.M. 1987) und Heinz Bude, Das Altern einer Generation. Die Jahrgänge 1938-1948 (Frankfurt a.M. 1995). 
erinnem, also Franz-Josef Strauß und Kiesinger damals, das weiß ich noch sebr genau, da habe ich am Radio gehangen, ich wollte eine Position dazu haben, was ich davon halten sollte, ob diese Bundeswebr nun bewaffnet werden soll und mit der atomaren Frage. Da habe ich angefangen, mich damit $z u$ beschäftigen. Aber auch noch relativ unpolitisch.

Das heißt moralisch?

Ja, moralisch. Und sehr mit einem existentialistischen Lebensgefübl. So, diese Welt ist sowieso nicht mehr zu retten, und diese Menschen werden das nicht aufhalten. $\mathrm{Ob}$ nun die Bundesrepublik Atomwaffen hat oder nicht, ist letztlich egal, das wird sowieso hier alles zu Bruch gehen. Also diese Vorstellung hatte ich ganz stark. Wenn diese Waffe erst mal erfunden ist, dann wird die auch in Aktion treten. Das entspricht sozusagen dem menschlichen Unvermögen und der menschlichen Unfäbigkeit, überhaupt ein vernünftiges Leben binzukriegen. Die moralische Inkompetenz des Menschen war eigentlich so ein Grundgefübl.

Wir lesen, wie Camilla Blisses Versuch, einen Ansatzpunkt zur Interpretation der Lage zu finden, scheitert. An die Aufrüstungsdebatte im Radio kann sie sich noch genau erinnern: Sie hört und lauscht, aber versteht nichts. Von einem großen Mißtrauen ist die Rede: Die Welt ist rettungslos verloren und der Mensch moralisch inkompetent. Camilla Blisse reagiert mit einer schwer gezügelten Verachtung auf eine Situation, die ihr verschlossen ist.

Man kann das „existentialistische Lebensgefüh]“ als Sperre gegen die Wahrnehmung der Modernitätsexplosion der 50 er Jahre ${ }^{19}$ verstehen. Sambaschritte und gelöster florealer Schwung, die Ersetzung des Rechtwinkligen durch das Bewegte, Bibi, Bulli, Babs - all diese Zeichen eines ebenso dynamischen wie amüsanten Aufbruchs nach einem schrecklichen Krieg gelten für die Jungakademikerin aus Bethel nichts, weil „diese Welt sowieso nicht mehr zu retten" ist.

Hier tritt uns mit Hayden White ${ }^{20}$ gesprochen das tragische Muster von Niedergang und Zerfall als Inversion des Komischen von Verwirrung und Rettung entgegen. Was heißt hier „sowieso“? Handelt es sie hier nur um ein jugendliches oder doch um ein generationsspezifisches Grundgefühl?

Zur Beantwortung dieser Frage bietet sich der Generationenvergleich an. Bei Christoph Westmeyer stellen sich die Dinge nämlich ganz anders dar.

Und in all dieser Zeit war mein Verhältnis in den SOer Jabren zu Deutschland ein gespaltenes. Ich hatte einen akademischen Lebrer, der war ein arger Deutschen-Hasser, und das hat mich sicherlich bewegt, oft das Land zu verteidigen. Andererseits hatte ich ein ambivalentes Verbältnis zu Deutschland. Ich fand, das Beste, was Deutschland widerfahren war, war die Teilung. Dadurch war es ein westliches Land geworden und kein Land der Mitte mehr, das seine Identität im Abstand zu Frankreich und zu England suchte, sondern jetzt eben sich als Teil des

19 Die illustriert Paul Maenz, Die 50er Jahre (Köln 1984).

20 Hayden White, Auch Klio dichtet oder Die Fiktion des Faktischen. Studien zur Tropologie des historischen Diskurses (Stuttgart 1986). 
Westens empfand. Wir waren eine Menge Probleme los, die sich aus dem Osten ergaben, die ganzen Krautjunker, die ja in der DDR beseitigt waren, das ganze Verständnis des Germanentums, alle Alternativen zum Westen, all dies war doch kein Thema mehr.

Mhm.

Die Grenzen waren geöffnet, und ich fand die Verwestlichung Deutschlands als etwas, wo das Land seine eigenen Talente erst konstruktiv entfalten würde. Und dann fand ich die Zeit faszinierend als eine Zeit des Neubaus. Manche meiner weltanschaulich engagierteren Kollegen redeten von Restauration und meinten damit etwas anderes. Sie meinten damit eine Haltung, die man als Antifaschismus bezeichnen könnte. Das war nicht einfach nur Antinationalsozialismus, das wäre ja einfach eine Zurückweisung gewesen, sondern auch eine positive Haltung, die von einer bestimmten Theorie für die Entstehung des Nationalsozialismus ausging und die zu schaffende Gesellschaft dann als Alternative dazu verstand.

$\mathrm{Mbm}$.

Diese Leuten haben kritisiert, daß Deutschland nach der Niederlage die Ansätze sozialistischer Art, die es in allen Parteien gab, zurückwies, den Nationalsozialismus nur ausgeklammert und nicht beseitigt hat, in gewisser Weise ja einfach vergaß. Und die haben das kritisiert. Ich war da anderer Meinung. Ich fand, das Beste, was wir tun können, also wenn einer Nazi war, zu sagen, komm, alle Sündigen, die anders sein wollen, denen wird vergeben. Wenn das deutsche Volk nur aus Antinazis bestehen darf, dann reicht das auch nicht so ganz hin. Wir werden also wobl mit Menschen dieses Land bauen müssen, die weniger als unbedingt vorzeigbar sind, aber immerhin, die wirklich also, die Schnittgrenze, wo für mich die Gemeinsamkeit aufhört, lag wesentlich weiter.

$\mathrm{Mhm}$.

Nämlich da eben, wo ich meine, das persönliche Engagement für das Regime bis hin zur Verfolgung anderer dazukam. Aber wenn einer einfach da mitgeschwommen ist, dachte ich, den muß man integrieren. Das war eine faszinierende Zeit des Neubaus, auch im wörtlichen Sinne. Ich sah diese Stadt entstehen.

Ja.

Vieles, was ich damals gut fand, finde ich heute schlecht. Wir haben damals die Stuckfassaden an den noch bestehenden Häusern kaputt geschlagen als Zeichen, als Geste, wie modern wir sind, und ich fand das großartig. Ich fand also, daß diese Bundesrepublik in vieler Hinsicht ein attraktives Land sei, mit Ausnabme dessen, was ich als Adenauer-Mief verstand, nämlich Tradierung einer kleinbürgerlichen Moral. Von daher war ich ein vehementer Kritiker der Adenauer-Regierungen, von denen ich meinte, die versuchen, Moralvorstellungen festzuschreiben, die die der zwanziger Jahre, ach, eigentlich beinah des Kaiserreichs sind. Und von daber war ich begeistert, als dann endlich die Regierung gezwungen war, zur Koalitionsregierung zu werden, als die Mehrbeit verloren ging.

In diesem biographischen Text gibt nicht heroische Tragik, sondern ironische Ambivalenz den Ton an. Da ist zunächst der Komplex von Teilung und Neubau, 
was das Deutschland nach dem Nationalsozialismus betrifft. Die Teilung ermöglichte die Entmischung der in seinen Augen gefährlichen deutschen Mischung aus westlicher Rationalität und östlicher Mystik, was zur Folge hatte, daß das Land "seine eigenen Talente“ überhaupt erst konstruktiv entfalten konnte. Teilung bedeutet für ihn nicht Strafe, sondern nimmt er als Bedingung für einen anderen Anfang nach 1945.

Man stößt hier auf das latente Strukturmuster von Entwertung und Anpassung. Entwertet wird alles, was war, damit die Anpassung an das gelingen kann, was ist. Christoph Westmeyer will keinen Schlußstrich ziehen, um etwas zu bewahren, sondern er nimmt hin, daß der Faden gerissen ist, um etwas ganz anderes zu probieren.

Dann haben wir im Text den Komplex von Destruktion und Fortschritt. Am Beginn dieser neuen Zeit stand eine Geste der Destruktion, die den unbedingten Willen zur Modernität unter Beweis stellen sollte. Man zerschlug die Stuckfassaden an den Häusern, die als Ornamente des Vergangenen galten, und errichtete, so könnte man fortfahren, neue Häuser, deren Oberfläche blank und glatt war. Das ist nicht nur der Ausdruck einer Erfahrung erhöhter Mobilität und Verstädterung, der Herausbildung neuer Freizeitstile und Konsumerwartungen, sondern auch das Bekenntnis zu einer Ästhetik des Bungalows, der seriellen Musik (Karlheinz Stockhausen) und des "Ich ohne Gewähr" (Ingeborg Bachmann). Christoph Westmeyer fand das ngroßartig“. Der Nihilist macht reinen Tisch mit der Geschichte, und der Positivist krempelt die Ärmel hoch. Die radikale Entwertung der schlechten Vergangenheit und die vollkommene Anpassung an die rasante $\mathrm{Ge}$ genwart gehören offenbar zusammen.

Schließlich ist da noch der Komplex aus moralischem Vergessen und funktionaler Einpassung. Alle, die mit anpacken wollen, sollen dazugehören. Zukunft ist wichtiger als Vergangenheit. Das ist ein unaufgeregter Nietzscheianismus, der ein historisches Abbruchunternehmen ohne Beispiel begleitet. Man wird dieses Land wohl mit Menschen bauen müssen, dic weniger als unbedingt vorzeigbar sind. $\mathrm{Da}$ helfen keine Anklage und keine Abrechnung. Die "Gleichgültigen neuen Stils" (David Riesman) können sich auch für einen "negativen Nationalismus" nicht begeistern.

Aber wer ist "Wir" ${ }^{\text {? }}$ Wer spricht? Von wo aus werden hier die 50er Jahre betrachtet? Für Camilla Blisse gibt es dieses „Wir“ überhaupt nicht. Für sie ist „diese Welt sowieso nicht mehr zu retten".

Ein zweites Paar von Textausschnitten fragt nun nach dem Herkunftskomplex dieser unterschiedlichen Haltungen zu den ${ }_{n} 50 \mathrm{er}$ Jahren ${ }^{*}$. Wie verstehen sie ihr Herkommen aus dem Nationalsozialismus? Welche Vergangenheit sind sie bereit, für sich zu akzeptieren?

Beginnen wir wieder mit Camilla Blisse.

Wie stand Ibr Vater zum Faschismus?

Mein Vater war überzeugter Nationalsozialist.

Ja. 
Ein ganz überzeugter Christ mit der Waffe. Er ist als Pfarrer freiwillig eingezogen, war schon bei der $S A$, was auch in zahlreichen Schriften und Briefen dokumentiert ist, seine Haltung zum Nationalsozialismus.

War Ihre Mutter anders?

Meine Mutter war in dieser Hinsicht halt eine typische Frau, das heißt, sie hat keine eigene Position entwickelt dazu, sie hat geglaubt, daß es so richtig sei, wie ihr Mann das gemacht hat, also sie hat gehofft und versucht, es so auch gut zu finden.

$\mathrm{Mbm}$.

Nehme ich jedenfalls an. Aber sie hat darüber nicht ein einziges Mal gesprochen. Das war das absolute Tabuthema. Und in diesem geschützten Raum Bethel ist der Nationalsozialismus politisch niemals ein Thema gewesen, in meiner gesamten Schulzeit ist das nicht einmal überhaupt berührt worden. Es war nur atmosphärisch halt so, daß Betbel eine karitative Einstellung zu den ganzen Problemen hatte, so daß auch sebr viele Nazis in Bethel untergebracht waren, und die da auch rumliefen, und so eine gewisse Berübrungsscheu den Menschen gegenüber bestand, aber ich nie genau wußte, warum. Ich war ja noch ziemlich klein und habe das erst binterher eigentlich alles rausgefunden, was da los war.

$\mathrm{Mhm}$.

Das war mir alles etwas unheimlich, aber ich habe als Kind viel weniger noch eine Abnung gehabt, was nun Nationalsozialismus war, ich habe mebr so sinnliche Erfabrungen mit dem Krieg selbst gehabt. Mit Bombenangriffen und Zerstörung von Städten, das hat mich, glaube ich, sebr, sebr tief beeindruckt, überhaupt die Zerstörungsleistung eines Krieges, obne damals zu fragen, wer eigentlich was hier $z \boldsymbol{u}$ verantworten hat. Erstmal überhaupt die Tatsache der Zerstörung, das war für mich eine solche Ungebeuerlichkeit, was ich nicht begreifen konnte, was das war, warum man das alles kaputt machen mußte.

Das hatte nichts mit einer Angezogenheit von großer Zerstörung zu tun, die ja wobl auch etwas Faszinierendes haben kann?

Nein, überhaupt nicht. Das war für mich wirklich die Hölle, wirklich die Hölle. Ich erinnere mich noch genau, als wir aus Polen kamen, das war 1944, da war gerade ein großer Angriff auf Berlin gewesen, und wir mußten da umsteigen von der Friedrichstraße zu einem anderen Babnhof und mußten durch diese kaputte Stadt laufen, und ich fand, das waren wirklich Höllenvorstellungen, so sieht die Hölle aus.

$\mathrm{Mbm}$.

Das war nachts, das vergesse ich nie. Auch andere Sachen. Zum Beispiel Bombenangriffe aus der Ferne haben wir mebrere erlebt, als wir evakuiert waren auf einem Dorf, wo man dann aus der Ferne so richtig sah, wie diese Bomben bei schönem Wetter 1945, diesem knallblauen Himmel, diese glitzernden Flugzenge, und dann die Bomben da unten rausflogen, und da sab man von weitem richtig, wie die Kirchtürme umkippten. Das sind so Erinnerungen. Auch wenn meine Mutter weinte, wegen der Gebäude auch, also auch. Ich weiß noch, wie sie sagte, diese schönen Kirchen. Das heißt also, auch so eine Art, von meiner Mutter ausgehend, Liebe zu der Kultur, die da zerstört wurde.

$M \mathrm{hm}$. 
$\mathrm{Zu}$ den Menschen, $z \boldsymbol{u}$ den anonymen Menschen und zu allem, was so eine Stadt an Gewordenem beinhaltet.

Glauben Sie, daß Ihre Mutter das ohne sie beide, die beiden Kinder ertragen bätte?

Nein, das glaube ich nicht. Das war eine emotional sehr enge Beziehung, das ist kaum vergleichbar, ich denke, daß diese Konstellation, diese Dreierkonstellation garnicht denkbar wäre unter anderen Bedingungen, unter Nicht-Kriegsbedingungen. Zumal in meiner Familie fast alle Männer gefallen waren, es sind keine übrig geblieben, die sind wirklich ausgerottet, und da war halt die Bindung aneinander und die Fürsorge füreinander und die Toleranz sehr, sehr einmalig, denke ich.

Wir werden mit einem harten und scharfen Bild des Vaters konfrontiert. Ein "ganz überzeugter " Täter, was in "zahlreichen " Quellen "dokumentiert" ist. Also kein Karrierist oder Mitläufer, sondern ein richtiger "Christ mit der Waffe“. Camilla Blisse spricht aus der Welt des evangelischen Pfarrhauses, die zwischen Glaube und Vaterland, Wille und Gehorsam, göttlicher Gnade und kriegerischer Bewährung liegt. Es handelt sich um einen Kontext ihrer Herkunft, der von der Mutter streng bewacht wird: „ein geschützter Raum“ und ein absolutes Tabu. Gleichwohl ist die atmosphärische Präsenz von etwas Unheimlichem mit Händen zu greifen. Der Vater wäre einer dieser herumschleichenden Nazis in Bethel gewesen, die schweigsam und geduckt sich dankbar für die karitative Aufnahme zeigen mußten. Da ist es schon besser, daß der Vater tot ist. Das doppelte Überzeugtsein, Christ und Nazi, macht ihn zu einer eigentümlich heroischen Gestalt, als sei er ein richtiger Held zur falschen Zeit gewesen. Vielleicht hätte aus ihm auch ein Martin Niemöller werden können, der vom U-Boot-Kommandanten des Ersten Weltkriegs zum militanten Pazifisten mutiert ist. Jedenfalls stellt sich die Frage, ob ein überzeugter Täter nicht letztlich besser als ein bloßer Mitläufer ist.

Das war eingerahmt von der "Hölle" des Krieges. Camilla Blisse rettet sich in eine anthropologische Empörung angesichts einer ${ }_{n}$ solchen Ungeheuerlichkeit ${ }^{\star}$. Es ist ein apokalyptisches Szenarium, wie sie als Vierjährige durch das zerstörte Berlin läuft. Die Erinnerung ist noch ganz plastisch vorhanden. Aber das Ganze hat auch eine abendländische Dimension: der "knallblaue Himmel" mit den "glitzernden Flugzeugen“. Die Mutter weist auf die „kippenden Kirchtürme“. Hat Gott die Menschen verlassen? Man fühlt sich an Hans Egon Holthusens Metapher vom "unbehausten Menschen" erinnert.

In die Hölle des Krieges ragt der Komplex einer bestimmten Bindung. Sie kommt aus einem Geschlecht „ausgerotteter Männer “. Das hat einen alttestamentarischen Ton, es klingen Assoziationen von Großartigkeit an. Aber ganz nah am Text stolpert man über diese Formulierungen: „Ausgerottet“ werden sollte doch ein anderes Volk?

Camilla Blisse schildert die Dreierkonstellation ihrer Herkunft so, daß man sich fragt, wer da eigentlich das Kind war. Die Mutter hätte es jedenfalls ohne die beiden Kinder nicht ertragen.

Über der durch „Bindung “, „Fürsorge“ und „Toleranz" zusammengehaltenen 
Restfamilie erhebt sich das Bild des Vaters mit seiner Überzeugung und seiner Waffe. Der harsche Ton und die stolze Geste der Verdammung, die unsere Erzählerin an den Tag legt, erinnern an die leere Stelle des Familienoberhaupts. Der heroische Vater paßt durchaus ins tragische Schema, und man muß sich fragen, wem sie die Treue hält und wessen Geschichte hier gelebt wird.

Bei Christoph Westmeyer kommt das Erbe des Nationalsozialismus ganz anders ins Spiel.

In dem Zusammenhang noch eine Frage, wie standen Sie zu den Lebrern, die jetzt wieder Lehrer waren? Konnten Sie sich von denen was sagen lassen?

Ja, ich habe ja solche und solche gehabt. Ich hatte ja echte Antinazi-Lebrer. Das hat mich als Kind in der Volksschule tief beeindruckt, mein Klassenlebrer war jemand, der vor den Augen der Kinder den Robrstock zerbrach und sagte, wir kommen obne aus. Also, das war nicht so, muß man auch wieder sagen. Von einem Nazi, Schmidtke, den haben wir nie wieder gesehen. Der ist allerdings in einer anderen Ecke von Aachen dann wieder Lebrer geworden.

$M \mathrm{hm}$.

Auf den hat man natürlich, habe ich natürlich einen tiefbleibenden $\mathrm{Ha} \beta$ gehabt, weil der uns damals verpfiffen hat. Nazi sein ist eine Sache und Leute verpfeifen eine andere. Das ist, wenn Sie so wollen, die doppelte Moral, die Moral für die Öffentlichkeit und die für das konkrete Leben.

Ja, das gibt es ja schon, die Sache mit dem berühmten Edelnazi, das war ja wobl eine Lebensrealität.

Ja, ja, der tut aber keinem was, nicht, während der andere den Leuten zum Anfassen was tut und nicht nur irgendwas Abstraktes, nicht. Juden hatten wir übrigens ab und zu mal zu Hause, solange es sowas noch gab, also bis '39, das war kein Problem bei uns. Ich muß übrigens noch eines hinzufügen. Konzentrationslager habe ich, der ich nun, weiß Gott, aus einer Familie komme, die den Nazis alles Böse zutraute, immer als brutale Arbeitslager verstanden. Daß es Vernichtungslager gab, hab' ich mich echt, ich hab' gedacht, die arbeiten die Leute zu Tode, nichts zu essen, Schläge und so, und was natürlich ganz schlimm ist alles. Aber die Idee der Vernichtungslager im industriellen Stil, das war eine echte Überraschung, und das hat mich tief getroffen. Und als ich das dann glauben mußte, das es so etwas gab wie Vermichtungslager, so ungefäbr ' 46 bab' ich das dann akzeptiert, daß das die Wabrheit ist, hatt' ich gedacht, Mensch als Deutscher wirst du nie wieder ein anständiges Leben fübren dürfen.

Mhm.

Das wird der Rest der Menschbeit dir nie verzeiben. Insoferm batte ich eigentlich mich damit abgefunden, soweit man das als junger Mensch tun kann, daß ich den Rest des Lebens als Mensch zweiter Klasse die Schulden meiner Väter abarbeiten muss.

Die Lakonie rettet Christoph Westmeyer nicht vor der Geschichte. An einigen Stellen kehrt die Realität wieder, der man nicht entkommen kann: Im Text zeugen der Lehrer Schmidtke, die Jahreszahlen 1939 und 1946 und der Begriff der Wahr- 
heit vom Alptraum der Geschichte. Es ist zu verfolgen, wie ein Ironiker des Nachkriegs mit der Realität des Nationalsozialismus ringt. Das Grundschema von Entwertung und Anpassung, das man nicht zu leicht und nicht zu schnell mit seelischer Derealisierung gleichsetzen sollte, funktioniert nicht vollständig. Da gibt es den „tiefbleibenden $\mathrm{Ha}^{\star}$ “ auf den Lehrer, der den Leuten was zum Anfassen tut. Der Unterschied zwischen diesem und dem Klassenlehrer, der vor den Augen der Kinder den Rohrstock zerbrach, macht deurlich, daß es durchaus einen Handlungsspielraum gab. Die Strategie der existentiellen Hinnahme und der situativen Relativierung geht da nicht auf. Außerdem läßt sich der Tatbestand des industriellen Massenmordes nicht leugnen. Der Ironiker erhebt nach 1945 den Anspruch auf ein nanständiges Leben“. Aber die Überwältigung durch eine verbrecherische Wirklichkeit läßt das nicht zu. Man muß das Geschehene hinnehmen und über das Ungeheuerliche schweigen.

Doch die Wirklichkeit läßt sich nicht zum Schweigen bringen: Da ist die Rede von der Differenz zwischen den zwei Moralen und der Differenz zwischen der eigenen Unschuld und den „Schulden meiner Väter“. Anders ausgedrückt: Es gibt eine Entscheidungsmöglichkeit zwischen ideologischer Parteinahme und konkretem Verbrechen; und es macht sich trotz ironischer Immunisierung eine tragische Schuld geltend.

Der Vergleich zwischen Camilla Blisse und Christoph Westmeyer läßt zwei Formen historischer Bindung deutlich werden: die ironische der Flakhelfer und Schülersoldaten und die tragische der Kriegskinder des Zweiten Weltkriegs. Wo die eine sich satirisch verfängt, ist die andere noch in der Anklage dem Angeklagten verfallen. Im ersten Fall kann man angesichts einer heillosen Geschichte nur davonkommen, im zweiten muß man sich einem fortgesetzten Schuldigwerden entgegenstellen. Für die Flakhelfer-Generation waren die 50er Jahre eine Zeit des biographischen Aufbruchs mit einsetzendem Wirtschaftswunder, beschwingten Linien, beginnendem Konsumoptimismus und sich festigender politischer Westbindung. Man fühlte sich befreit zum Vergessen und disponiert zur Einpassung. Aber auch unfähig zur politischen Leidenschaft und zur moralischen Empörung. Für die Generation der Kriegskinder hingegen waren die 50er Jahre eine Zeit bleierner Restauration und autoritärer Sozialisation. Sie fühlten sich „in der Grube (Rolf Dieter Brinkmann) und belastet mit Eltern, die die Last ihrer eigenen Geschichte nicht tragen konnten. Sie waren noch zu jung für die Wahrnehmung des Neuen und neigen deshalb im nachhinein dazu, nur die Restauration des Alten zu sehen.

Vergleicht man von heute aus die Wirkungsgeschichte der 45er- mit der der 68er-Generation, dann sieht es so aus, als hätte das skeptische Schweigen der Schülersoldaten über die hilflose Empörung der Kriegskinder den Sieg davongetragen. So wie man in der Zeitgeschichtsforschung jetzt die Modernität der 50er Jahre entdeckt ${ }^{21}$ und die 60er Jahre nicht allein von ihrem Ende her beurteilt ${ }^{22}$,

${ }^{21}$ Axel Schildt, Amold Sywottek (Hrsg.), Modernisierung im Wiederaufbau. Die Westdeutsche Gesellschaft der 50er Jahre (Bonn 1993). 
wird im kollektiven Bewußtsein der Bundesrepublik die Anschlußfähigkeit der Flakhelfer-Generation unterdessen höher eingeschätzt als die Protestfähigkeit der 68er-Generation. Das gilt sogar für "Liebhaber der Revolution“ selbst. Als sich mit der Bundestagswahl von 1998 für die 68er-Generation in Gestalt des "rotgrünen Bündnisses" eine zweite Chance bot, waren ihre Repräsentanten an der Macht sofort bereit, den Heroismus vergangener Tage zu vergessen und sich zum Pragmatismus der Bundesrepublik zu bekennen. Nach der Einigung des Landes scheint der Rückgriff auf die existentielle Skepsis verläßlicher als die Disposition eines moralischen Alarmismus.

22 Axel Schildt, Detlef Siegfried, Karl Christian Lammers (Hrsg.), Dynamische Zeiten. Die 60er Jahre in den beiden deutschen Gesellschaften (Hamburg 2000). 